九州大学学術情報リポジトリ

Kyushu University Institutional Repository

\title{
Estimation of fatigue damage in holed composite laminates using an embedded FBG sensor
}

Yashiro, Shigeki

Department of Mechanical Engineering, Shizuoka University

Okabe, Tomonaga

Department of Aerospace Engineering, Tohoku University

http://hdl. handle. net/2324/4476058

出版情報: Composites Part A: Applied Science and Manufacturing. 42 (12), pp.1962-1969, 201109-03. Elsevier

バージョン :

権利関係 : 


\title{
Estimation of fatigue damage in holed composite laminates using an embedded FBG
} sensor

S. Yashiro ${ }^{\mathrm{a},{ }^{*}}$ and T. Okabe ${ }^{\mathrm{b}}$

${ }^{\text {a }}$ Department of Mechanical Engineering, Shizuoka University

3-5-1 Johoku, Naka-ku, Hamamatsu, 432-8561, Japan

${ }^{\mathrm{b}}$ Department of Aerospace Engineering, Tohoku University

6-6-01 Aoba-yama, Aoba-ku, Sendai, 980-8579, Japan

* Corresponding author: Tel: +81-53-478-1026; Fax: +81-53-478-1026.

E-mail address: tsyashi@ipc.shizuoka.ac.jp (S. Yashiro)

\begin{abstract}
This study investigated damage identification in holed CFRP laminates under cyclic loading by using an embedded fiber Bragg grating (FBG) sensor. Ply cracks and delamination extended near the hole with an increasing number of cycles, and the reflection spectrum from the FBG sensor was distorted. Moreover, debonding growth of the FBG sensor was observed. This study then estimated laminate damage pattern from reflection spectra and investigated the influence of the sensor debonding on damage identification. The debonding length was estimated from the spectrum simulated with a given debonding length and was successfully identified only when an appropriate damage pattern was assumed. Moreover, greater debonding induced invalid damage-pattern estimates, even if the debonding length was given in the estimation. The damage identification for simulations and for experiments required half of the intact gage section. These estimates indicated that information on the damage pattern disappeared from the spectrum shape because of debonding.
\end{abstract}

Keywords: A. Polymer-matrix composites (PMCs), B. Fatigue, B. Delamination, Inverse analysis. 


\section{Introduction}

Carbon fiber reinforced plastics (CFRPs) have been applied to primary structural components because of their beneficial mechanical properties (e.g., high specific strength, high specific modulus, and high fatigue limit). However, microscopic damage occurs due to cyclic loading in composite laminates. In particular, matrix cracks and delaminations accumulate near stress concentrations, and the damage pattern is complicated [1]. Moreover, the extension of fatigue damage causes strength degradation.

Structural health monitoring (SHM) systems that detect damage extension are useful for improving structural reliability. Fiber Bragg grating (FBG) sensors have been used to accurately measure strain and temperature. FBGs have also been applied to detect non-uniform strain distribution. Peters et al. [2,3] and Botsis et al. [4] embedded FBG sensors in various non-homogeneous strain fields and confirmed their response by analytical modeling. Takeda and colleagues [5,6] applied the sensitivity of FBG sensors to non-uniform strain fields to the detection of transverse cracks and delamination in CFRP laminates. Garrett [7] used embedded FBG sensor networks to evaluate local residual post-impact strain of woven composite laminates. The authors $[8,9]$ monitored the extension of complex tensile damage near stress concentrations using FBG sensors, and analyzed the mechanisms of damage detection. Sorensen et al. [10] measured non-uniform strains due to mode-I delamination by optical low-coherence reflectometry and the inverse scattering method, and identified bridging tractions in double cantilever beam composite specimens. Takeda [11] and Tsuda [12] employed FBG sensors for Lamb wave detection to evaluate impact damage in CFRP laminates.

Our previous study [13] applied embedded FBG sensors to detect damage extension in holed CFRP cross-ply laminates under cyclic loading. The stacking configuration was cross-ply $\left[0_{2} / 90_{2}\right] \mathrm{s}$; the specimen was $30 \mathrm{~mm}$ wide and had a hole with a diameter of $5 \mathrm{~mm}$ at the center. The specimen was subjected to cyclic loading, where the maximum stress was 260 $\mathrm{MPa}(0.4 \%$ strain $)$ and the stress ratio was 0.1 . This stress level was lower than that of the first damage generation in static loading. The FBG sensor debonded from the matrix due to cyclic 
loading (Fig. 1), but did not debond under static loading. A narrowband spectrum was measured after debonding (Fig. 2) in fatigue tests, since debonding induced a constant longitudinal strain along the FBG sensor. Thus, information on the laminate (damage) was lost from the reflection spectrum. Here, the longitudinal strain extracted from the embedded FBG coincided with the host material, although the FBG sensor was coated with polyimide.

However, most previous studies [5-12] detected damage by completely bonded FBG sensors, and debonding of the embedded sensor has not been analyzed. As with cyclic loading [13], progressive debonding of the FBG sensor from epoxy resin was indicated after sufficient exposure to moisture [14]. Therefore, the effect of debonding on damage monitoring should be clarified, considering long-term durability.

In this study, fatigue damage of the holed composite laminate was numerically estimated from reflection spectra of a debonded FBG sensor, and the applicability of the damage estimation scheme was investigated versus the debonding length. First, inverse analysis of damage identification [15] was extended to consider debonding of the embedded FBG sensor. Damage of the laminate and debonding were then estimated from reflection spectra of both simulations and experiments. Estimation accuracy was analyzed considering the debonding length.

\section{Inverse analysis of damage identification}

The damage patterns of composite laminates were estimated from the reflection spectra of an embedded FBG sensor independently of the physical damage extension process. This inverse analysis required an analytical model that reproduced an arbitrary damage pattern, and finite-element analysis that could control the damaged region by cohesive elements was then developed. Since this finite-element model did not analyze debonding of the embedded optical fiber, shear-lag analysis was performed to calculate the strain distribution in the debonded optical fiber. Section 2.1 describes the analytical procedure to calculate the reflection spectrum of the debonded FBG sensor embedded in a holed composite laminate with an arbitrary damage pattern. 
Section 2.2 describes the inverse analysis of damage identification. Design variables representing the laminate damage pattern were optimized by minimizing errors between the target spectrum and the calculated one.

\subsection{Analytical model}

In order to analyze the reflection spectrum considering the damage in the laminate, the strain distribution of the embedded optical fiber was calculated by finite-element analysis, and cohesive elements were used to represent various cracks in the laminate. The cracking condition of all cohesive elements was controlled to correspond to the design variables described later.

The CFRP cross-ply laminate depicted in Fig. 2 was studied. A $0.76 \mathrm{~mm}$-thick laminate was modeled for each ply, and damage with ply size was represented by cohesive elements. Figure 3 illustrates a $1 / 8$ model considering symmetry. The model was divided into two layers of $0^{\circ}$ ply and $90^{\circ}$ ply, and four-node isoparametric Mindlin plate elements were applied to these layers to investigate out-of-plane deformation. Furthermore, an optical fiber modeled by two-node truss elements was introduced into the $0^{\circ}$ layer along the fiber $(x)$ direction. The embedding position in the transverse $(y)$ direction was less than $1.5 \mathrm{~mm}$ and was varied to correspond to the specimens. Since an optical fiber hardly carried loads except the longitudinal load, truss elements were sufficient for analyzing the strain distribution.

This analysis considered splits, transverse cracks, and delamination; cohesive elements were then built into the position so that damage would occur. The relationship between traction $T$ and relative displacement $\Delta$ was expressed as follows [16].

$$
T_{i}=\frac{s}{1-s} \frac{\Delta_{i}}{\Delta_{i c}} \tau_{i \max } \quad(i=n, t, b)
$$

Here, the subscript $n$ denotes the cracking mode of normal tension, $t$ denotes the in-plane shear, and $b$ denotes the out-of-plane shear; $\tau_{i \max }$ is the maximum stress, and $\Delta_{i c}(i=n, t, b)$ is the critical relative displacement in each cracking mode. The critical relative displacement is calculated from the critical energy release rate $G_{i c}(i=\mathrm{I}, \mathrm{II}, \mathrm{III})$. 


$$
\Delta_{n c}=\frac{2 G_{\mathrm{I} c}}{\tau_{n \max } s_{i n i}}, \quad \Delta_{t c}=\frac{2 G_{\mathrm{II} c}}{\tau_{t \max } s_{i n i}}, \quad \Delta_{b c}=\frac{2 G_{\mathrm{III} c}}{\tau_{b \max } s_{i n i}}
$$

The variable $s$ in Eq. (1) represents all cracking conditions from intact cohesion to no cohesion; the cohesive element is equivalent to a penalty element when s is equal to an initial value $s_{i n i}(=0.999$ in this study), and becomes a crack surface when $s$ is zero. As described in the following section, an arbitrary laminate damage pattern was developed by controlling the distribution of parameter $s$ as a function of design variables. The strain distribution along the optical fiber was obtained by solving the virtual work principle.

Since this finite-element analysis did not consider debonding of the embedded optical fiber, one-dimensional shear-lag analysis [13] was conducted to analyze the longitudinal strain distribution of the debonded optical fiber from that of the laminate. The optical fiber was assumed to be debonded from the position nearest to the hole and to have constant longitudinal strain in the debonded part. If the polyimide coating is assumed to transfer stress to the glass cladding, the shear stress of the coating is given by

$$
\tau=\frac{G\left(w_{L A M}-w_{f}\right)}{R_{c}-R_{f}},
$$

where $G$ is the shear modulus of the coating, wLAM $_{\text {is }}$ the displacement of the laminate at the embedding position, $w_{f}$ is the displacement of the glass cladding, $R_{c}$ is the radius of the coating, and $R_{f}$ is the radius of the glass cladding. The following equation is derived by considering stress transfer to the cladding by the coating.

$$
E \frac{\mathrm{d}^{2} w_{f}}{\mathrm{~d} z^{2}}=-\frac{2 G\left(w_{L A M}-w_{f}\right)}{R_{f}\left(R_{c}-R_{f}\right)}
$$

Here, $E$ is the Young's modulus of the glass fiber. In order to solve Eq. (4), two boundary conditions are imposed: (1) the strain at the debonding tip, $\varepsilon_{D}$, is continuous, and (2) the displacement of the optical fiber is equal to that at the end of the laminate. The strain in debonding, $\varepsilon_{D}$, is converged taking into account the boundary conditions to obtain the displacement distribution of the optical fiber $w f$. First, $\varepsilon_{D}$ was assumed to be a certain value $(\varepsilon D 0)$, and $w_{f}$ for a given debonding length $d_{f}$ was obtained from Eq. (4) and boundary 
conditions. $\varepsilon_{D}$ was then calculated based on the geometry of the optical fiber.

$$
\varepsilon_{D}=\frac{1}{d_{f}}\left\{\varepsilon_{L A M} L-\left(w_{f}^{e}-w_{f}^{t}\right)\right\},
$$

where $\varepsilon_{L A M}$ is the applied strain for the model, $L$ is the length of the model, and superscripts $e$ and $t$ for $w_{f}$ denote the model end and the debonding tip. The calculated $\varepsilon_{D}$ is used as the assumed value $\varepsilon D 0$, and the same procedure is iterated until $\varepsilon D$ converges sufficiently. The longitudinal strain distribution of the optical fiber is finally calculated by the obtained displacement distribution $w f$.

The corresponding reflection spectrum is numerically analyzed from the strain distribution of the optical fiber $\varepsilon_{f}(x)$. An FBG sensor has periodic change in the refractive index of the core of the optical fiber, and reverse mode coupling can be generated in a waveguide. For an FBG sensor embedded in a laminate, the grating period $\Lambda$ and the effective refractive index of the core $n_{\text {eff }}$ are obtained as linear functions of the longitudinal strain $\varepsilon f[5,8]$.

$$
\begin{aligned}
& \Lambda(x)=\left(1+\varepsilon_{f}(x)\right) \Lambda_{0} \\
& n_{e f f}(x)=n_{0}-\frac{n_{0}^{3}}{2}\left\{p_{12}-v_{f}\left(p_{11}+p_{12}\right)\right\} \varepsilon_{f}(x)
\end{aligned}
$$

Here, $\Lambda_{0}$ is the initial value for the grating period, $n_{0}$ is the initial value for the effective refractive index, $v_{f}$ is Poisson's ratio of the optical fiber, and $p_{11}$ and $p_{12}$ are the strain-optic coefficients where indices 1 and 2 denote the longitudinal direction and transverse direction of the optical fiber. The gage section with those sensor profiles is divided into many segments, and the grating period and the effective refractive index are assumed to be uniform in each segment. Mode coupling between the forward wave and the backward wave in each segment is then numerically calculated by the transfer matrix method $[8,17]$.

Thus, a combination of finite-element analysis for the laminate with a controlled damage pattern, shear-lag analysis for the debonded optical fiber, and optical analysis is used to calculate the reflection spectrum of the FBG sensor, considering the damage pattern in the laminate. 


\subsection{Damage identification method}

The damage pattern in the laminate was estimated from the reflection spectrum of the embedded FBG sensor. Parameters to be estimated in the inverse analysis (i.e., design variables) are the size and shape of the delamination, and the size of transverse cracks. A damage pattern corresponding to a set of the design variables is temporarily developed in the analytical model, and the reflection spectrum is calculated. The calculated spectrum then numerically approximate the target (input) spectrum by optimizing the damage pattern. This damage identification involves an optimization problem that minimizes errors between the shape of the input spectrum and the estimated one.

$$
\text { Minimize: } F(\mathbf{d})=\sum_{m=0}^{100}\left\{a_{m}-\widetilde{a}_{m}(\mathbf{d})\right\}^{2}
$$

\section{Design variables: $\mathbf{d}$}

The reflection spectrum is represented by a Fourier series to quantitatively evaluate its shape, and $a_{m}$ and $\tilde{a}_{m}$ are the $m$ th Fourier coefficients for the input spectrum and the estimated one. The estimated reflection spectrum $\tilde{a}_{m}$ includes the influence of damage and debonding through the strain distribution of the gage section. The combination of the design variables that minimizes $F(\mathbf{d})$ is defined as the identified result.

Table 1 lists the physical meanings of the design variables, and Fig. 4 schematically illustrates the design variables, which are defined considering physical damage extension near the hole. The damaged zone and damage process zone are represented by setting the distribution of the residual strength parameter $s$ of cohesive elements as functions of the design variables $\mathbf{d}$. The shape of the delamination is approximated by the following equation in Cartesian coordinates $\xi-\eta$ whose origin is $(x, y)=(2.0,2.5)$.

$$
\left(\frac{\xi}{d_{d 1}}\right)^{\alpha}+\left(\frac{\eta}{d_{d 2}}\right)^{\beta} \leq 1, \quad \xi \geq 0, \quad \eta \geq 0
$$

Here, $d_{d 1}$ is the half length of the major axis of delamination, and $d_{d 2}$ is the half length of the minor axis of delamination; $\alpha$ and $\beta$ define the delamination shape. Equation (9) can draw various curved lines within $0 \leq \xi \leq d_{d 1}$ and $0 \leq \eta \leq d_{d 2}$ and draws an ellipse when $\alpha=\beta=2$. The 
cohesive elements for delamination are completely damaged in the area expressed by Eq. (9), and the residual strength parameter $s$ of these elements is set to zero. The damage process zone where $0<s<\operatorname{sini}$ is assumed to have a shape similar to that of a completely damaged zone, and its half length of the major axis is represented by design variable $d d 3$. The distribution of the residual strength parameter in the damage process zone is expressed by a power law of the distance from the delamination tip.

$$
s=s_{\text {ini }} r^{p}, \quad 0 \leq r \leq 1
$$

Here, the normalized distance $r$ is zero at the delamination tip and becomes unity at the tip of the process zone along a straight line connecting the origin of the $\xi-\eta$ coordinates and an arbitrary point. Design variable $p$ denotes the recovery of the cohesive stiffness. Thus, delamination is expressed by the six parameters $d_{d 1}, d_{d 2}, d_{d 3}, \alpha, \beta$, and $p$.

The distance from the hole edge to the farthest transverse crack is defined as design variable $d_{c 1}$. Under cyclic loading, transverse cracks gradually extend in the transverse direction (Fig. 2). The lengths of all the transverse cracks are assumed to be identical (design variable $d_{c 2}$ ).

A previous study [8] demonstrated that splits in the $0^{\circ}$ ply have little effect on the reflection spectrum of the FBG sensor in the $0^{\circ}$ ply. Thus, the splitting is assumed to be the same size as the delamination process zone, since delamination near the hole extends from the tips of the splits.

$$
d_{s}=d_{d 3}+2.0
$$

Furthermore, debonding of the FBG sensor occurs from the hole edge with the highest stress, and the debonding length is expressed by design variable $d_{f}$. Accordingly, the design variable for all the damage types is $\mathbf{d}=\left\{d_{\mathrm{d} 1}, d_{d 2}, d_{d 3}, \alpha, \beta, p, d_{c 1}, d_{c 2}, d_{f}\right\}^{T}$.

The damage identification procedure is as follows.

1. Material properties and initial values of the design variables $\mathbf{d}$ are given.

2. The damage pattern corresponding to the set of design variables is developed in the finite-element model by controlling the parameter $s$ of cohesive elements. 
3. The strain field of the laminate is analyzed by finite-element analysis.

4. The strain distribution of the optical fiber is calculated for debonding length $d_{f}$ by shear-lag analysis.

5. The reflection spectrum is analyzed by the transfer matrix method considering the strain distribution of the FBG sensor. Here, the obtained spectrum differs from the target.

6. The values of the design variables are modified based on a mathematical optimization technique (Fletcher-Powell method with the golden-section linear search) to minimize the objective function $F(\mathbf{d})$.

7. Steps 2 to 6 are repeated for the new design variables until $F(\mathbf{d})$ has a minimum value. Here, $F(\mathbf{d})$ contains many local minimum solutions, where the corresponding damage pattern is invalid.

8. In order to obtain the global minimum solution, the tunneling algorithm [18] is applied at each local minimum solution. Steps 2 to 7 are repeated until no smaller value of $F(\mathbf{d})$ can be found.

The optimization problem had nine design variables, and calculation costs were high for all variables. Therefore, before optimizing all variables, a solution for each damage type was sought to obtain presumable initial values for all variables. This study estimated items in the following order: (1) debonding, (2) transverse cracks, (3) delamination, and (4) all the variables.

\section{Estimated results and discussion}

Tables 2 and 3 list the material properties and parameters of the cohesive elements used in this analysis. Uniform tensile stress was loaded at the longitudinal edge of the model, and the residual stress due to temperature change $(\Delta T=-100 \mathrm{~K})$ during molding was also considered. Table 4 lists the optical properties for the embedded FBG sensor.

Sections 3.1 and 3.2 estimated damage and debonding from simulated results with given debonding lengths to investigate their sensitivity. Damage identification is demonstrated for experiments in section 3.3. 


\subsection{Estimating debonding length}

Reflection spectra corresponding to given debonding lengths were calculated for the simulated damage state at $N=10^{4}$ (Fig. 5), where $N$ is the number of cycles. The debonding length was estimated from these reflection spectra. First, the design variables, except for $d_{f}$, were fixed at values that represented the damage pattern depicted in Fig. 5a. The estimated debonding length is plotted in Fig. 6a ( $\mathbf{a})$. The estimation error relative to the given length was within the size of a single mesh of the analysis model for debonding smaller than the gage length $(12.5 \mathrm{~mm})$, and the debonding length was accurately estimated. The debonding length was then estimated when no damage was assumed in the laminate. The result plotted in Fig. 6a (०) greatly differed from the given length.

Figure $6 \mathrm{~b}$ plots the wavelength at the maximum intensity of the reflection spectrum, which approximately corresponds to the strain in the gage section. The estimated wavelength coincided with that of the target spectrum when the correct damage pattern was assumed. However, when no damage occurred, the estimated wavelength was shorter than that of the target when debonding was within the gage length. This result indicated that the average strain in the gage section could not be reproduced by debonding alone. Therefore, an appropriate damage pattern was required to estimate the debonding length.

\subsection{Damage pattern estimation}

This section investigates the effect of debonding on estimation accuracy for the damage pattern. The damage pattern depicted in Fig. 5a was estimated from the simulated reflection spectrum considering a given debonding length, where debonding was fixed at the given length during optimization. Figure 7 depicts the estimated results for debonding with a length of $5 \mathrm{~mm}$ and that with a length of $10 \mathrm{~mm}$. The estimated reflection spectrum agreed well with the input in both cases. When debonding was $5 \mathrm{~mm}$ long (Fig. 7a), slight delamination and transverse cracks appeared in the estimation and agreed well with the damage pattern depicted in Fig. 5a. However, a larger damaged region than that in Fig. 5a was estimated for greater 
debonding (Fig. 7b).

Two clear peaks appeared in the input spectrum when the debonding length was $40 \%$ to $60 \%$ of the gage length as depicted in Fig. $5 \mathrm{~b}$. The intensity of the peak in the longer wavelength increased with increasing debonding length. Therefore, this peak corresponded to the constant strain due to debonding. The peak in the shorter wavelength corresponded to the strain distribution of the laminate and contained information on the damage in the laminate. In the reflection spectrum in Fig. $7 \mathrm{~b}$, the peak at the shorter wavelength $(1553 \mathrm{~nm})$ lost intensity due to significant debonding, and the spectrum had little information on the laminate. In this case, the input spectrum represented only the strain in the debonded gage section, and the damage could not be estimated.

Thus, the accuracy of damage pattern estimation decreased with increasing debonding length, since the embedded FBG sensor lost sensitivity to the strain distribution. The damage pattern depicted in Fig. 5a was estimated for some debonding lengths, and the result revealed that half of the gage section was required to identify the damage in the laminate.

\subsection{Damage identification for experiment results}

Damage patterns observed in the experiment were identified from the reflection spectra measured in the fatigue tests (Fig. 2b). First, the estimated debonding length was plotted in Fig. 8. The reflection spectrum for $N<10^{4}$ contained information on the laminate, since the estimated debonding length was less than the gage length of the FBG sensor. However, inappropriate results of the damage pattern should be obtained for $N \geq 10^{4}$ because of significant debonding over the gage section.

Figure 9a illustrates the estimated results of the reflection spectrum at $N=10^{3}$. Although two peaks were not reproduced in the estimate, a step appeared at the shorter peak wavelength of the experiment $(1553.3 \mathrm{~nm})$, and the spectrum width agreed. Here, estimated design variables except for debonding length were presented by a schematic illustration of the damage pattern (Fig. 9b). There was no delamination, but a few small transverse cracks were estimated near the hole. This damage pattern almost agreed with the observation. There was 
no splitting, since splitting was directly linked to the size of the delamination process zone $d d 3$. Similarly, the estimated damage pattern agreed with the observation for $N=2 \times 10^{3}$ and $5 \times 10^{3}$.

Figure 10a illustrates the results estimated of the reflection spectrum at $N=10^{4}$. The estimated spectrum reproduced the narrowband peak of the measured spectrum, and the peak wavelength coincided with the input. However, compared with the observation, greater delamination and more transverse cracks were obtained (Fig. 10b) where the estimated debonding length was $24.0 \mathrm{~mm}$. Similar to Fig. 7b, the lack of information on the laminate in the reflection spectrum resulted in poor estimation. Reasonable damage states could not be obtained by inverse analysis for $N \geq 10^{4}$ because of significant debonding.

The spectrum width corresponded to the range of the strain in the gage section. The spectrum width obtained by inverse analysis coincided with the measured one for a few cycles $\left(N<10^{4}\right)$, although forward analysis [13] predicted a narrowband spectrum with a FWHM of $0.3 \mathrm{~nm}$. Therefore, with a few cycles $\left(N<10^{4}\right)$, the actual strain distribution was well represented by inverse analysis rather than forward analysis. Meanwhile, a narrowband spectrum (e.g., Fig. 10a) was produced by a constant strain, and the constant strain could be reproduced by both debonding of the optical fiber and delamination near the hole. Since the only factor for a narrowband spectrum was the strain in the gage section, the debonding length varied in accordance with the change in the global strain in the laminate induced by the laminate damage pattern, especially by delamination size. This fact indicated the existence of some patterns that provided a single narrowband spectrum, and the uniqueness condition of the inverse problem was violated. Therefore, inaccurate estimates would be obtained by inverse analysis with significant debonding. The above discussion suggested that the debonding length in the fatigue tests was approximated by the dashed line in Fig. 8, which was obtained by using the results of inverse analysis for $N<10^{4}$ and those of forward analysis for $N \geq 10^{4}$.

\section{Conclusions}

This study estimated the damage states in CFRP cross-ply laminates with a hole under 
cyclic loading by inverse analysis of the reflection spectrum of an embedded FBG sensor. Debonding of the FBG sensor was observed in the fatigue tests. Shear-lag analysis for calculating the strain distribution of the debonded optical fiber was combined with damage analysis using cohesive elements and optical analysis for an FBG sensor to calculate the reflection spectrum. The damage pattern in the laminate and the debonding length were estimated from the reflection spectrum by an optimization technique with numerical analyses. The sensitivity of the debonding length and of the damage pattern in the laminate was investigated by applying inverse analysis to simulation results with given debonding lengths. Furthermore, the damage pattern was estimated from experiment results of fatigue tests. The conclusions are summarized below.

1. An appropriate damage pattern was required for accurately estimating the debonding length. The strain in the gage section could not be reproduced by debonding alone.

2. Half of the intact gage section was essential to estimate the damage pattern in the laminate. The reflection spectrum of the FBG sensor became a single peak and lost information on the strain distribution of the laminate when the debonding area exceeded the gage section.

3. A constant strain that yielded a narrowband spectrum could be reproduced by both debonding of the optical fiber and delamination near the hole. The damage-pattern estimation using FBG sensors was therefore applied in experiments with little debonding.

\section{Acknowledgement}

S. Y. acknowledges the support of the Mazda Foundation under a research grant. S. Y. also acknowledges support from the Ministry of Education, Culture, Sports, Science and Technology of Japan under a Grant-in-Aid for Scientific Research (No. 22760524). T. O. acknowledges the support of the Ministry of Education, Culture, Sports, Science and Technology of Japan under Grants-in-Aid for Scientific Research (No. 22360352, 20360379, 22360044). T. O. also acknowledges the support of the New Energy and Industrial Technology Development Organization (NEDO) (Project No. P08024). 


\section{References}

[1] Spearing SM, Beaumont PWR. Fatigue damage mechanics of composite materials. I: Experimental measurement of damage and post-fatigue properties. Compos Sci Technol 1992; 44(2):159-168.

[2] Peters K, Pattis P, Botsis J, Giaccari P. Experimental verification of response of embedded optical fiber Bragg grating sensors in non-homogeneous strain fields. Opt Laser Eng 2000; 33(2):107-119.

[3] Peters K, Studer M, Botsis J, Iocco A, Limberger H, Salathé R. Embedded optical fiber Bragg grating sensor in a nonuniform strain field: Measurements and simulations. Exp Mech 2001; 41(1):19-28.

[4] Botsis J, Humbert L, Colpo F, Giaccari P. Embedded fiber Bragg grating sensor for internal strain measurements in polymeric materials. Opt Laser Eng 2005; 43(3-5):491-510.

[5] Okabe Y, Yashiro S, Kosaka T, Takeda N. Detection of transverse cracks in CFRP composites using embedded fiber Bragg grating sensors. Smart Mater Struct 2000; $9(6): 832-838$

[6] Takeda S, Okabea Y, Takeda N. Delamination detection in CFRP laminates with embedded small-diameter fiber Bragg grating sensors. Compos Part A 2002; 33(7):971-980.

[7] Garrett RC, Peters KJ, Zikry MA. In-situ impact-induced damage assessment of woven composite laminates through a fibre Bragg grating sensor network. Aeronaut J 2009; 113(1144):357-370.

[8] Yashiro S, Takeda N, Okabe T, Sekine H. A new approach to predicting multiple damage states in composite laminates with embedded FBG sensors. Compos Sci Technol 2005; 65(3-4):659-667.

[9] Yashiro S, Okabe T, Takeda N. Monitoring damage in holed CFRP laminates with chirped FBG sensors. Int J Solid Struct 2007; 44(2):603-613.

[10] Sorensen L, Botsis J, Gmur T, Cugnoni J. Delamination detection and characterisation 
of bridging tractions using long FBG optical sensors. Compos Part A 2007; 38(10):2087-2096.

[11] Takeda N, Okabe Y, Kuwahara J, Kojima S, Ogisu T. Development of smart composite structures with small-diameter fiber Bragg grating sensors for damage detection: Quantitative evaluation of delamination length in CFRP laminates using Lamb wave sensing. Compos Sci Technol 2005; 65(15-16):2575-2587.

[12] Tsuda H. Ultrasound and damage detection in CFRP using fiber Bragg grating sensors. Compos Sci Technol 2006; 66(5):676-683.

[13] Okabe T, Yashiro S. Damage detection in holed composite laminates using an embedded FBG sensor. Compos Part A, submitted for publication.

[14] Karalekas D, Cugnoni J, Botsis J. Monitoring of hygrothermal ageing effects in an epoxy resin using FBG sensor: A methodological study. Compos Sci Technol 2009; 69(3-4):507-514.

[15] Yashiro S, Okabe T, Takeda N. Damage identification in a holed CFRP laminate using a chirped fiber Bragg grating sensor. Compos Sci Technol 2007; 67(2):286-295.

[16] Geubelle PH, Baylor JS. Impact-induced delamination of composites: a 2D simulation. Compos Part B 1998; 29(5):589-602.

[17] Huang S, LeBlanc M, Ohn MM, Measures RM. Bragg intragrating structural sensing. Appl Opt 1995; 34(22):5003-5009.

[18] Levy AV, Montalvo A. The tunneling algorithm for the global minimization of functions. SIAM J Sci Comput 1985; 6(1):15-29.

\section{Figure captions}

Fig. 1 Debonding between the FBG sensor and the matrix observed in a fatigue test [13]. The FBG sensor was embedded in a $0^{\circ}$ ply along the fiber direction and positioned near a hole.

Fig. 2 Experiment results of the damage pattern in the cross-ply laminate and the reflection spectrum of the embedded FBG sensor [13]. The maximum loading was $0.4 \%$ strain at 
positions away from the hole, and the stress ratio was 0.1 .

Fig. 3 Layer-wise finite-element model for the cross-ply laminate with an open hole and with an embedded FBG sensor.

Fig. 4 Definition of the design variables to represent the damage pattern near the hole.

Fig. 5 Simulated fatigue damage at $N=10^{4}$ and corresponding reflection spectrum with given debonding lengths.

Fig. 6 Estimation of the debonding length from the simulated spectrum with given debonding lengths. Inappropriate damage pattern (no damage) induced large error, since the peak wavelength (i.e., average strain) could not be reproduced by debonding alone.

Fig. 7 Damage identification results from the simulated reflection spectrum with given debonding lengths, where the correct damage pattern was depicted in Fig. 5. Significant debonding resulted in wrong estimates due to the single-peak spectrum of debonding.

Fig. 8 Debonding length estimated from the reflection spectra measured in the fatigue tests, where the number in legends denotes the specimen number. The length predicted by damage extension simulation [13] is also plotted.

Fig. 9 Estimated results from the reflection spectrum measured in the fatigue tests $\left(N=10^{3}\right)$, where the estimated debonding length was $2 \mathrm{~mm}$. The damage pattern with small transverse cracks and no delamination was estimated and agreed with the experiment.

Fig. 10 Estimated results from the reflection spectrum measured in the fatigue tests $\left(N=10^{4}\right)$. Large delamination and many transverse cracks, which disagreed with the experiment, were estimated, since the estimated debonding length $(24.0 \mathrm{~mm})$ exceeded the gage length. 
Table 1 Summary of the design variables to represent the damage pattern.

$d_{d 1} \quad$ Half-length of the major axis for the delamination

$d d 2 \quad$ Half-length of the minor axis for the delamination

$d d 3 \quad$ Half-length of the major axis for the damage process zone of delamination

$\alpha \quad$ Parameter to determine the shape of the delamination in Eq. (9)

$\beta \quad$ Parameter to determine the shape of the delamination in Eq. (9)

$p \quad$ Parameter governing the recovery of the residual strength $s$ in the damage process

$p \quad$ zone, Eq. (10)

$d_{c 1} \quad$ The distance from the hole edge to the transverse crack farthest from the hole

$d_{c 2} \quad$ The length of transverse cracks

$d_{f} \quad$ Debonding length of the embedded optical fiber. 
Table 2 Properties of materials used in the analysis.

\section{For CFRP}

Longitudinal Young's modulus (GPa)

Transverse Young's modulus (GPa)

In-plane shear modulus (GPa)

Out-of-plane shear modulus (GPa)

In-plane Poisson's ratio

Out-of-plane Poisson's ratio

Longitudinal thermal expansion coefficient $\left(\times 10^{-6} \mathrm{~K}^{-1}\right)$

Transverse thermal expansion coefficient $\left(\times 10^{-6} \mathrm{~K}^{-1}\right)$

For optical fiber

Young's modulus of glass $(\mathrm{GPa})$

Young's modulus of coating (GPa)

Poisson's ratio of glass

0.16

Thermal expansion coefficient of glass $\left(\times 10^{-6} \mathrm{~K}^{-1}\right)$

0.5

Thermal expansion coefficient of coating $\left(\times 10^{-6} \mathrm{~K}^{-1}\right)$

60 
Table 3 Properties of cohesive elements.

For splits and transverse cracks

In-plane tensile strength (MPa) 76

In-plane shear strength (MPa) 100

Out-of-plane shear strength (MPa) 100

Mode I critical energy release rate $\left(\mathrm{J} / \mathrm{m}^{2}\right) \quad 200$

Mode II critical energy release rate $\left(\mathrm{J} / \mathrm{m}^{2}\right) \quad 600$

Mode III critical energy release rate $\left(\mathrm{J} / \mathrm{m}^{2}\right) \quad 600$

For delamination

In-plane tensile strength (MPa) $\quad 30$

In-plane shear strength (MPa) 60

Out-of-plane shear strength (MPa) 60

Mode I critical energy release rate $\left(\mathrm{J} / \mathrm{m}^{2}\right) \quad 300$

Mode II critical energy release rate $\left(\mathrm{J} / \mathrm{m}^{2}\right) \quad 1000$

Mode III critical energy release rate $\left(\mathrm{J} / \mathrm{m}^{2}\right) \quad 1000$ 
Table 4 Optical properties of the FBG sensor.

Gage length (mm)

Initial center wavelength (nm)

Initial refractive index of the core

Depth of index modulation

Strain-optic coefficient $p_{11}$

Strain-optic coefficient $p_{12}$
12.5

1550.28

1.4490

$4.0 \times 10^{-4}$

0.113

0.252 


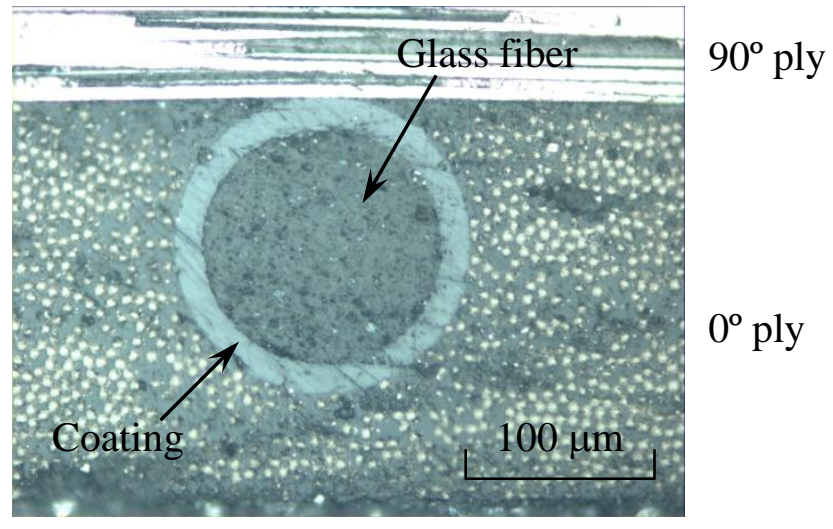

(a) Cross-section away from the hole (without damage)

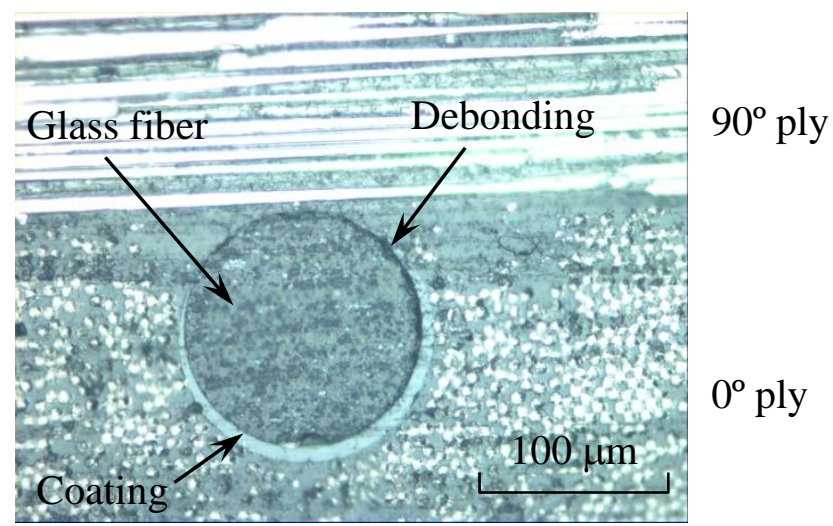

(b) Cross-section near the hole $\left(N=10^{6}\right)$

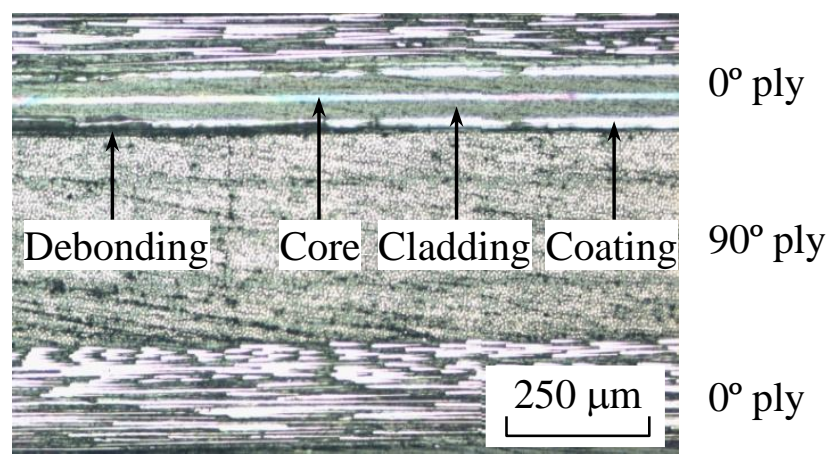

(c) Cross-section near the hole parallel to the optical fiber $\left(N=10^{6}\right)$

Fig. 1 Debonding between the FBG sensor and the matrix observed in a fatigue test [13]. The FBG sensor was embedded in a $0^{\circ}$ ply along the fiber direction and positioned near a hole. 
Fig. 2
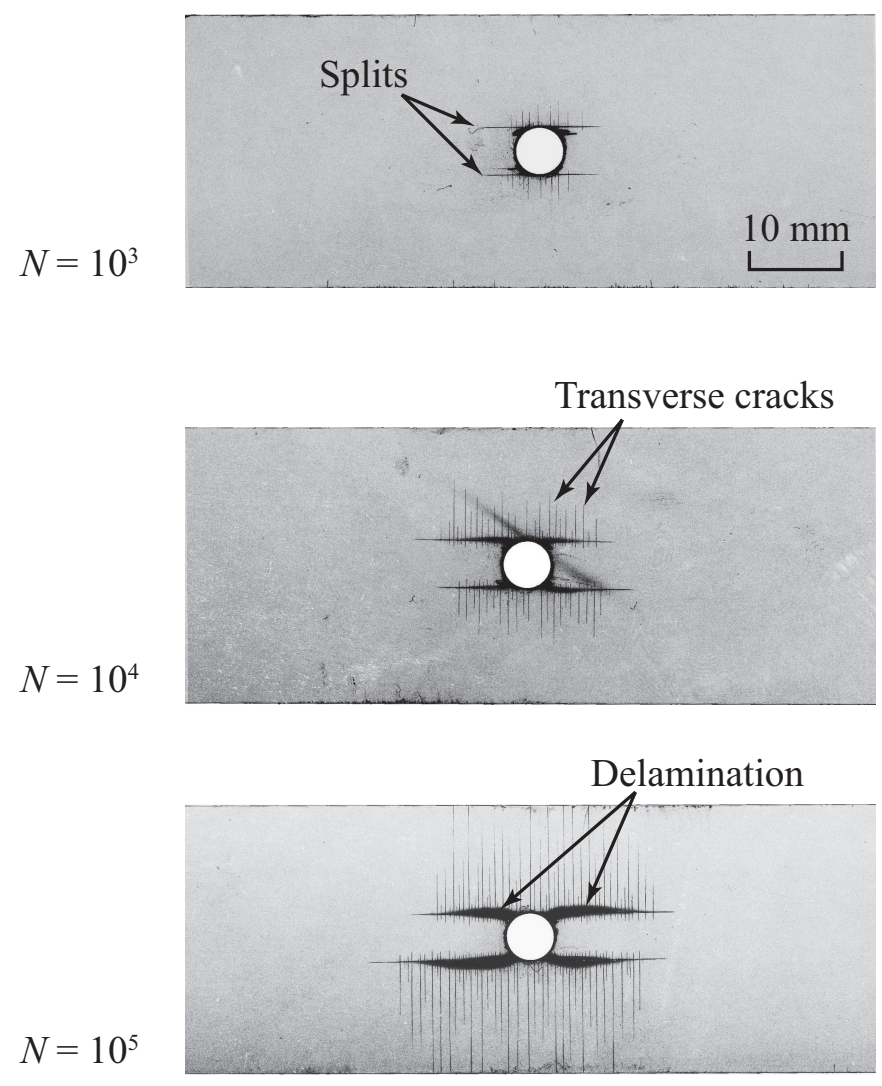

(a) Soft X-ray photograph

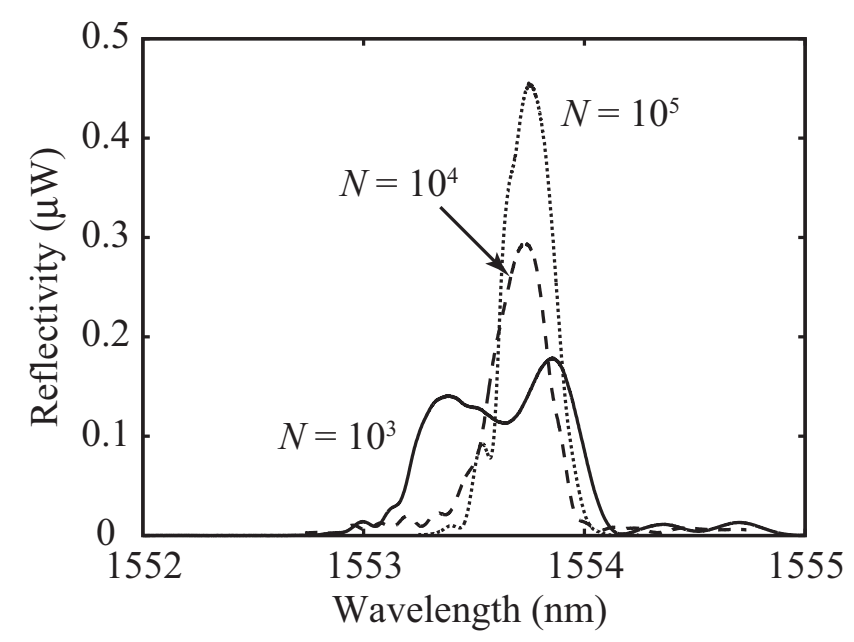

(b) Reflection spectrum

Fig. 2 Experiment results of the damage pattern in the cross-ply laminate and the reflection spectrum of the embedded FBG sensor [13]. The maximum loading was $0.4 \%$ strain at positions away from the hole, and the stress ratio was 0.1 . 
Fig. 3

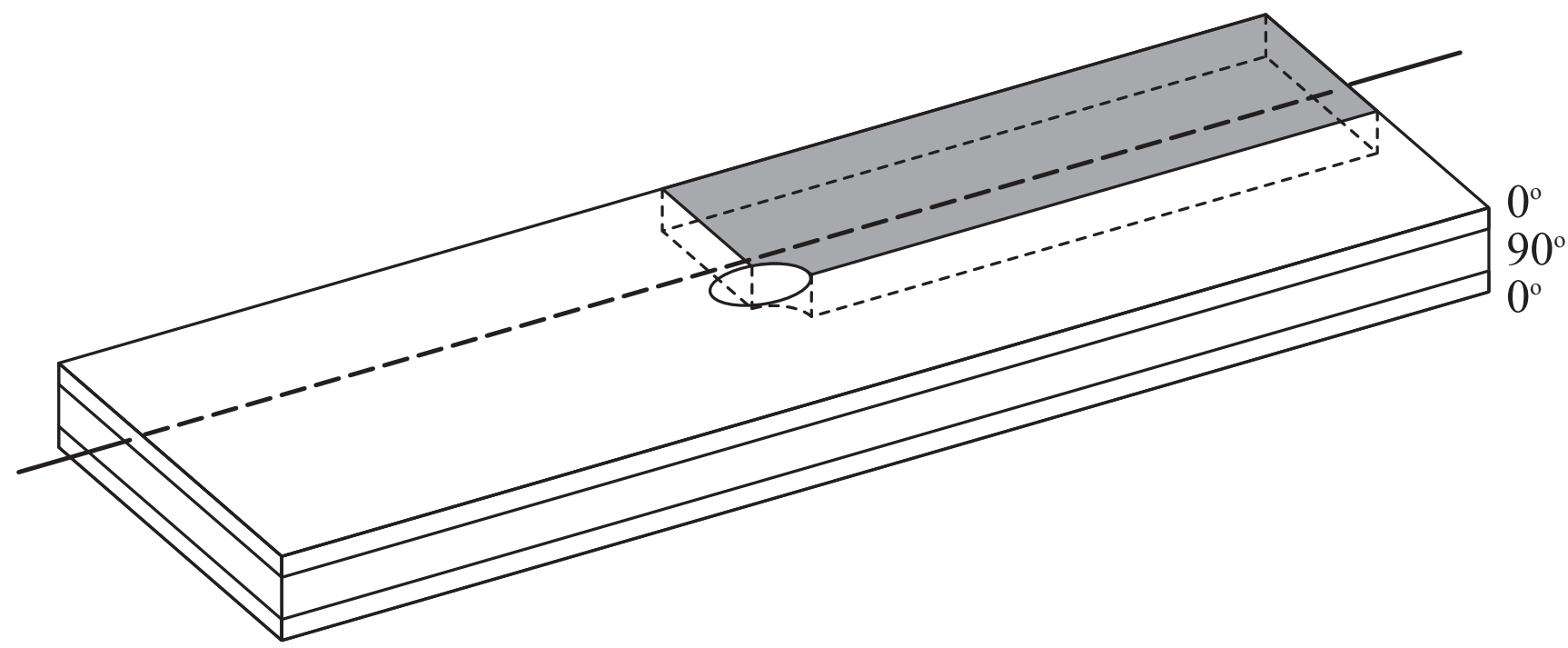

(a) Schematic

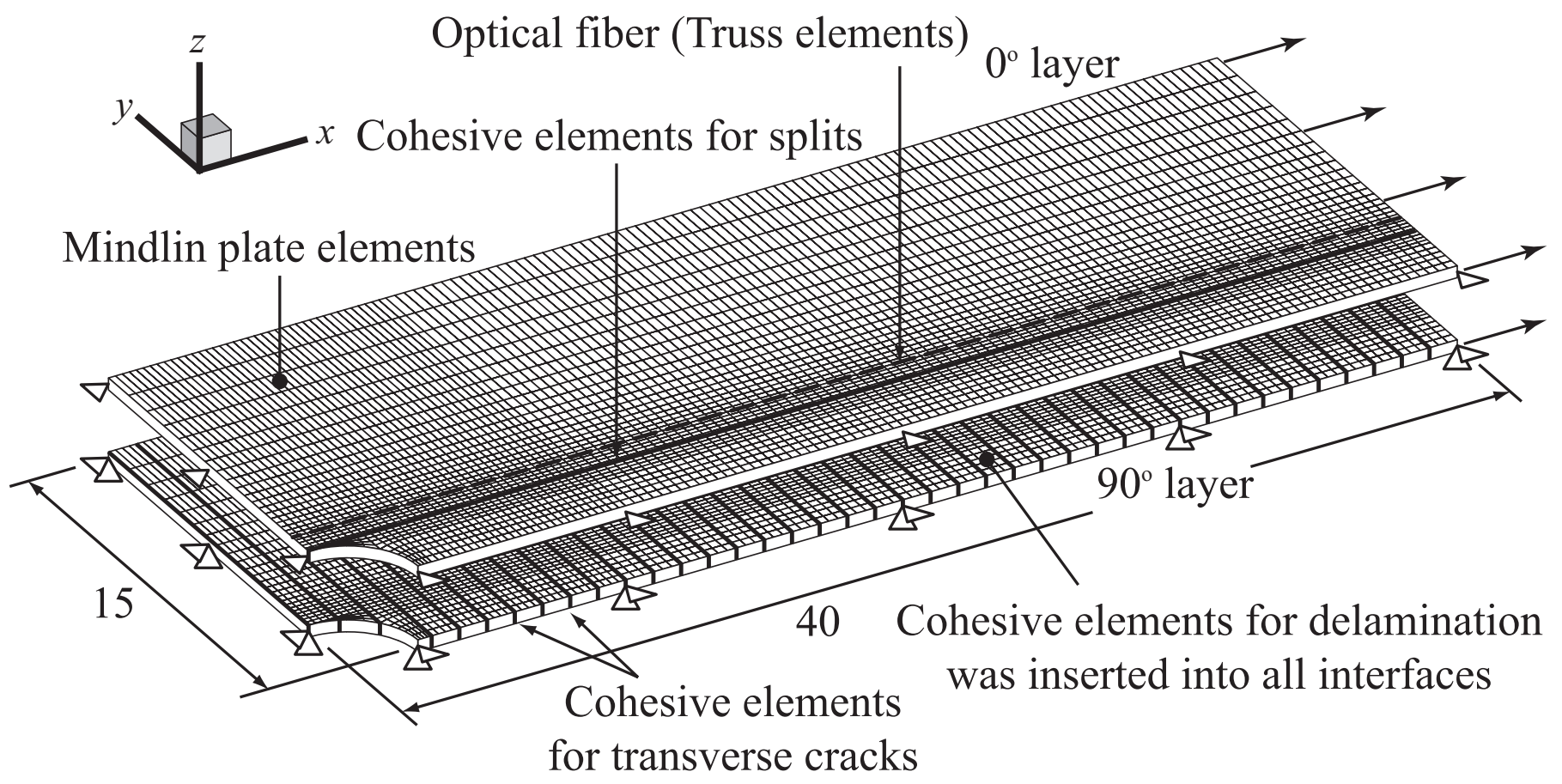

(b) Finite-element mesh

Fig. 3 Layer-wise finite-element model of the cross-ply laminate with an open hole and with an embedded FBG sensor. 
Fig. 4

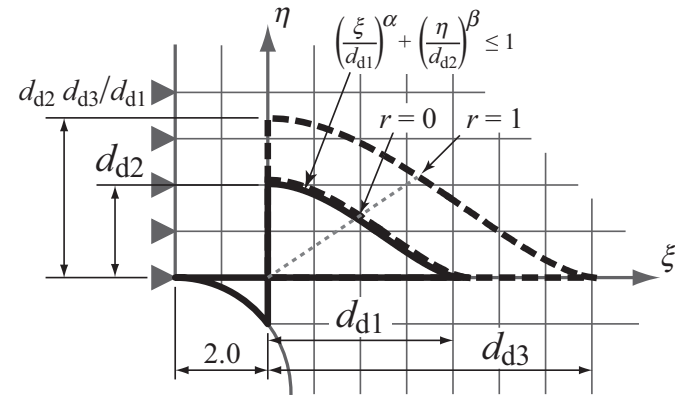

(a) Delamination

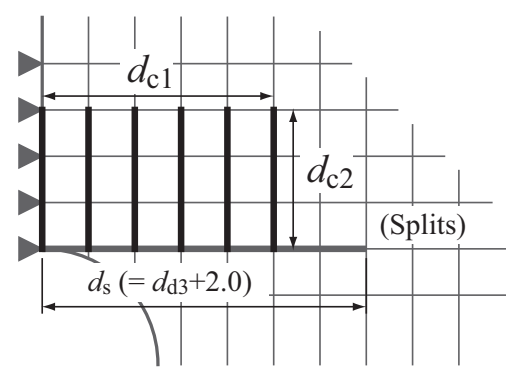

(c) Transverse cracks

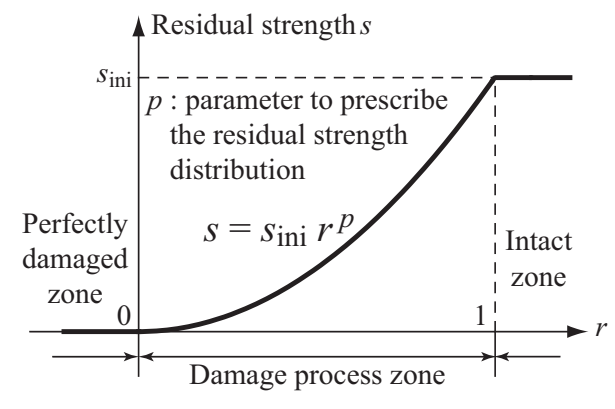

(b) Distribution of residual strength

- Perfectly damaged zone - - Damage process zone

Fig. 4 Definition of the design variables to represent the damage pattern near the hole. 
Fig. 5

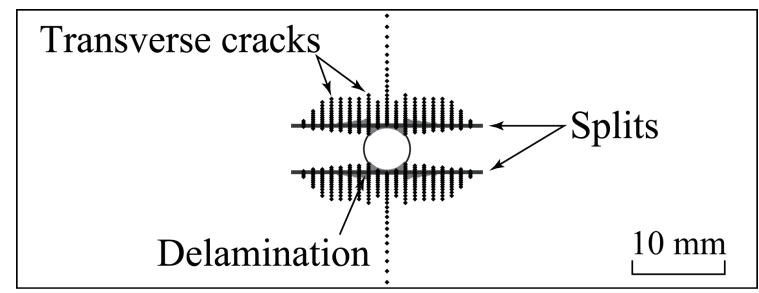

(a) Damage pattern

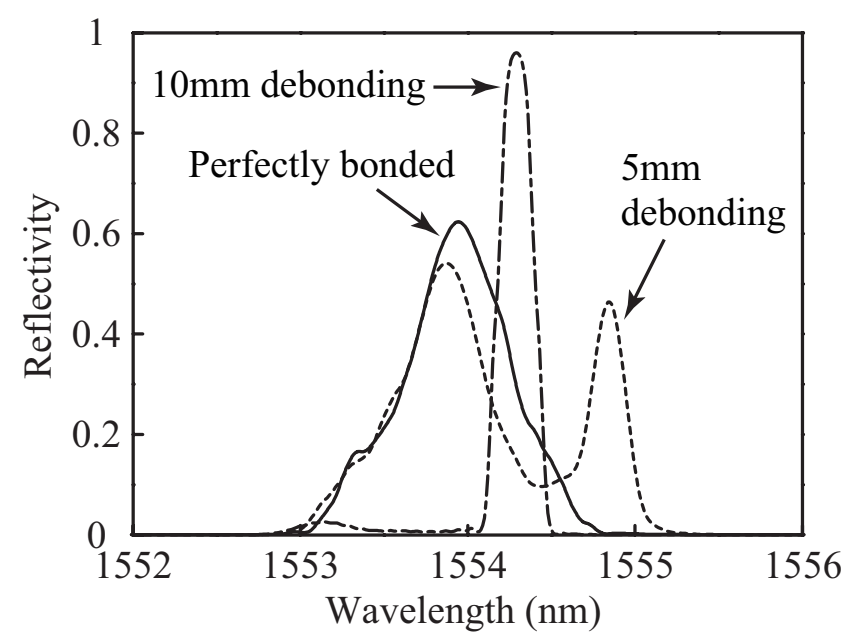

(b) Reflection spectrum

Fig. 5 Simulated fatigue damage at $N=10^{4}$ and corresponding reflection spectrum with given debonding lengths. 
Fig. 6

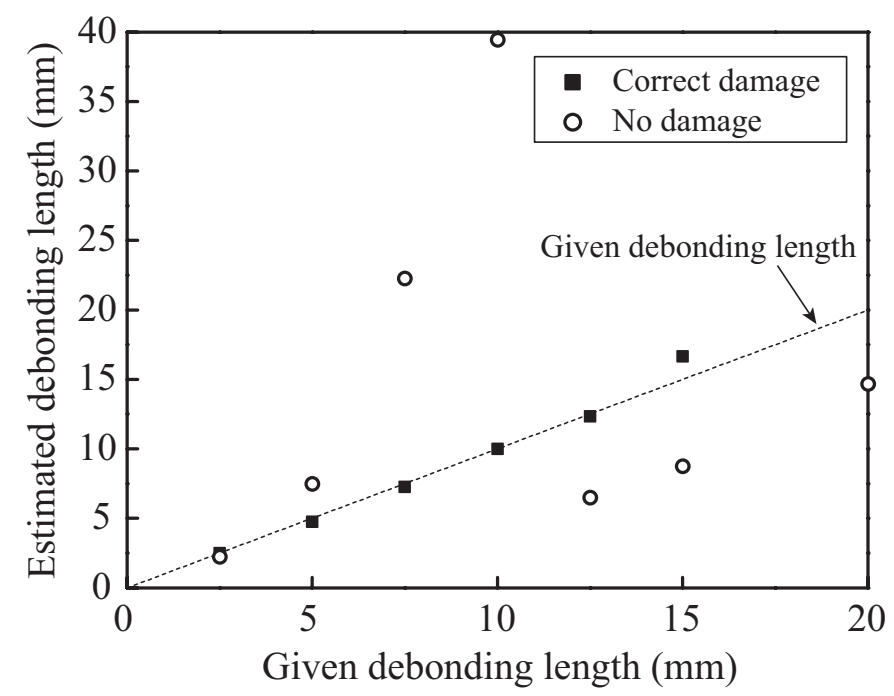

(a) Debonding length

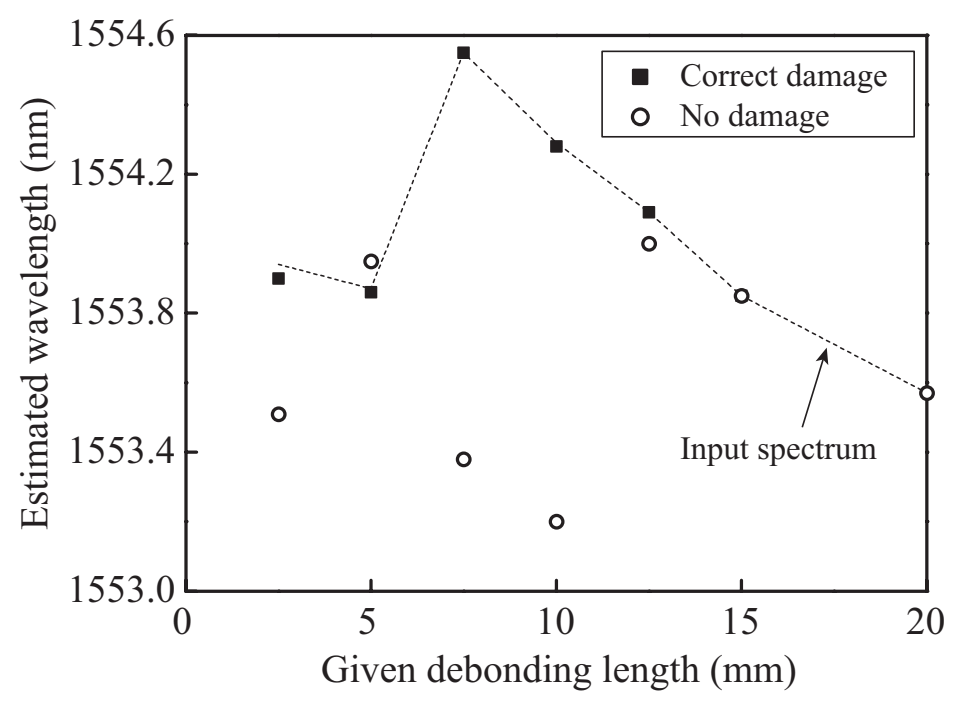

(b) Wavelength at the maximum reflectivity

Fig. 6 Estimation of the debonding length from the simulated spectrum with given debonding lengths. Inappropriate damage pattern (no damage) induced large error, since the peak wavelength (i.e., average strain) could not be reproduced by debonding alone. 
Fig. 7
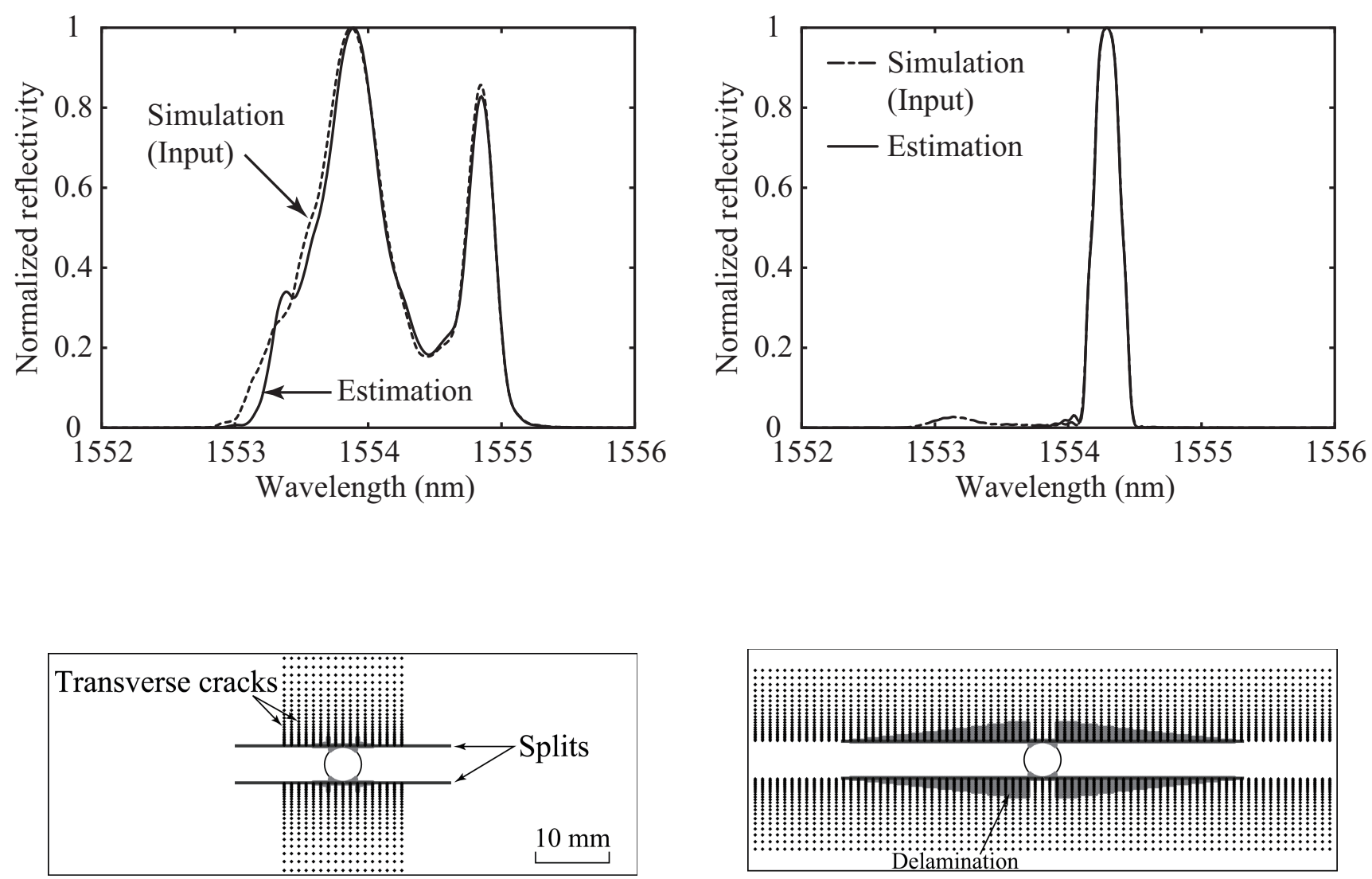

(a) $5 \mathrm{~mm}$ long debonding

(b) $10 \mathrm{~mm}$ long debonding

Fig. 7 Damage identification results from the simulated reflection spectrum with given debonding lengths, where the correct damage pattern was depicted in Fig. 5. Significant debonding resulted in wrong estimates due to the single-peak spectrum of debonding. 
Fig. 8

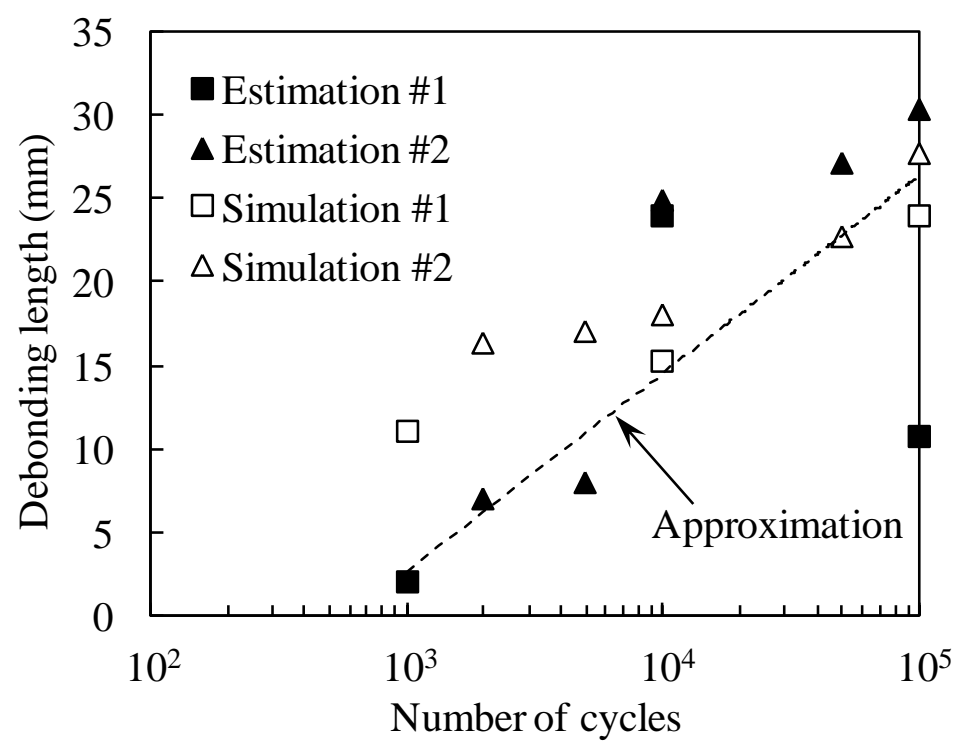

Fig. 8 Debonding length estimated from the reflection spectra measured in the fatigue tests, where the number in legends denotes the specimen number. The length predicted by damage extension simulation [13] is also plotted. 
Fig. 9

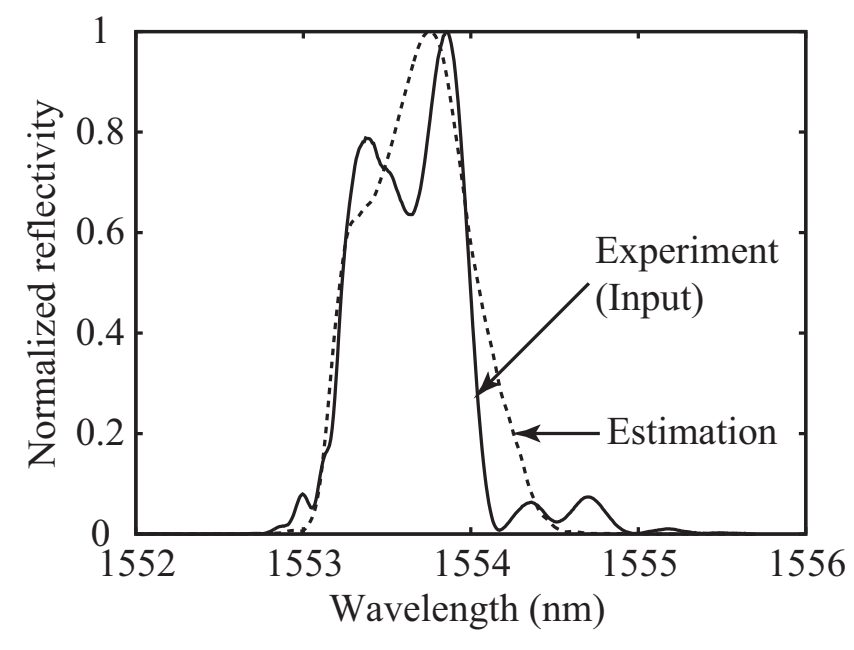

(a) Reflection spectrum
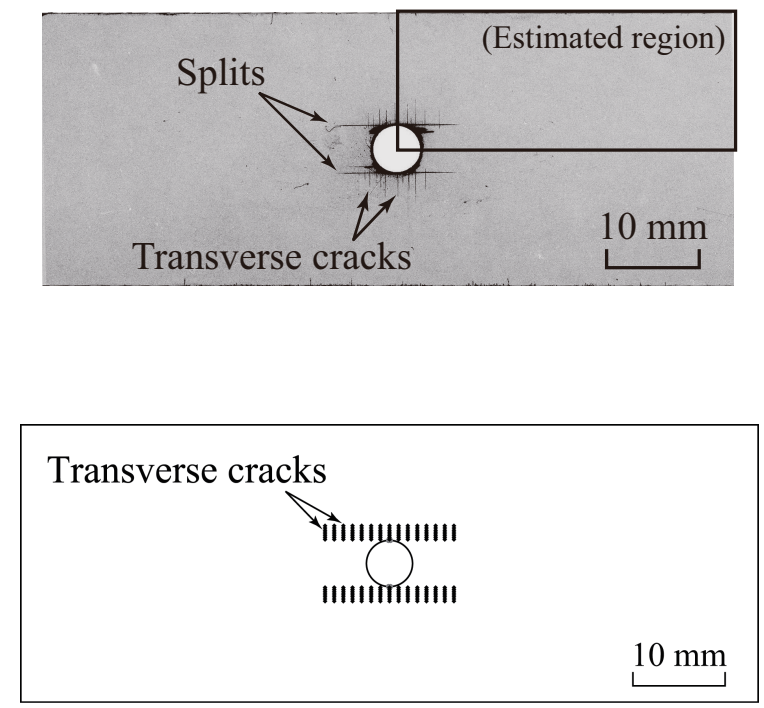

(b) Damage pattern

Fig. 9 Estimated results from the reflection spectrum measured in the fatigue tests $\left(N=10^{3}\right)$, where the estimated debonding length was $2 \mathrm{~mm}$. The damage pattern with small transverse cracks and no delamination was estimated and agreed with the experiment. 


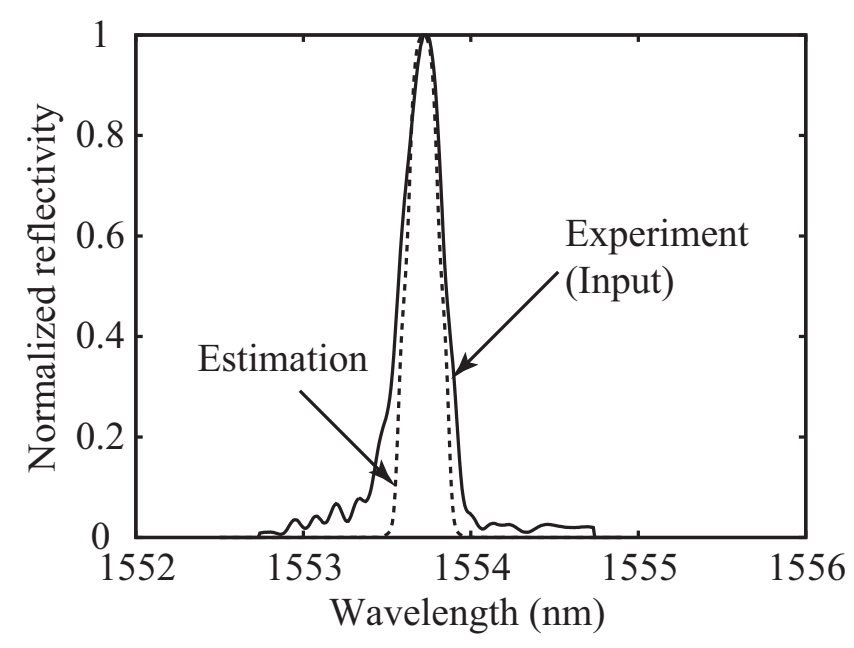

(a) Reflection spectrum
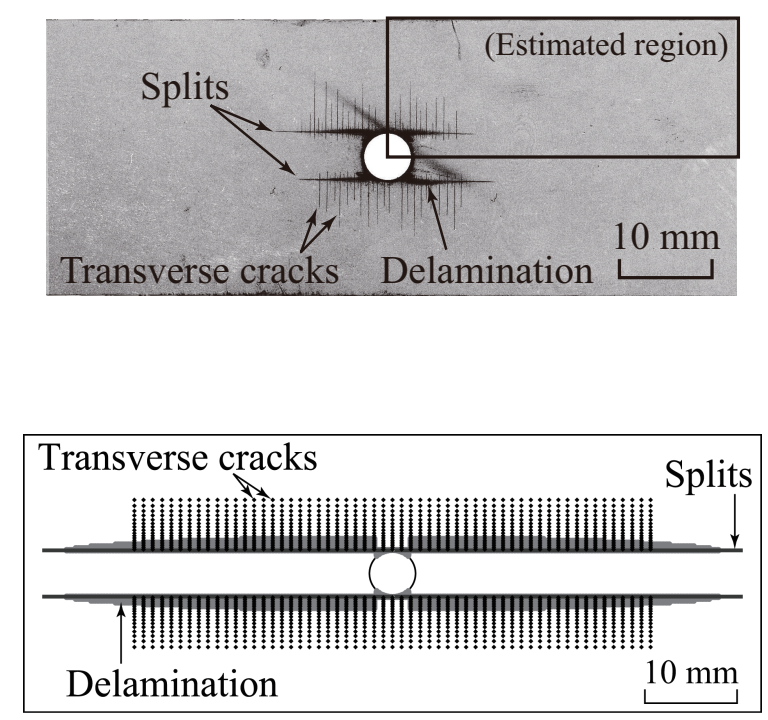

(b) Damage pattern

Fig. 10 Estimated results from the reflection spectrum measured in the fatigue tests $\left(N=10^{4}\right)$. Large delamination and many transverse cracks, which disagreed with the experiment, were estimated, since the estimated debonding length $(24.0 \mathrm{~mm})$ exceeded the gage length. 